\title{
Universal Scaling Relations in Strongly Anisotropic Materials
}

\author{
M. B. Hastings ${ }^{1, *}$ and C. Mudry ${ }^{2}$ \\ ${ }^{1}$ Center for Nonlinear Studies and Theoretical Division, Los Alamos National Laboratory, Los Alamos, New Mexico 87545, USA \\ ${ }^{2}$ Condensed Matter Theory Group, Paul Scherrer Institut, CH-5232 Villigen PSI, Switzerland
}

(Received 15 June 2005; published 20 January 2006)

\begin{abstract}
We consider the critical temperature in strongly anisotropic antiferromagnetic materials, with weak coupling between stacked planes, in order to determine the interplane coupling constant from experimentally measured susceptibilities. We present theoretical arguments for a universal relation between interplane coupling and susceptibility shown numerically by Yasuda et al. [Phys. Rev. Lett. 94, 217201 (2005)]. We predict a more general scaling function if the system is close to a quantum critical point, a similar relation for other susceptibilities than considered in Yasuda et al., and the validity of these relations for more general phase transitions.
\end{abstract}

DOI: 10.1103/PhysRevLett.96.027215

PACS numbers: 75.10.Jm, 75.30.Kz, 05.10.Cc

Many materials display at low temperatures strongly spatially anisotropic responses to magnetic or electronic probes. This fact has motivated the theoretical study of low dimensional quantum systems in their own right. Solving one- or two-dimensional quantum systems can be useful to understand intermediary regimes of temperature in which fluctuations are dominated by the subsystem of lower dimensionality. Three dimensionality is effectively restored once the temperature is lowered below the lowest energy scale characterizing the anisotropy.

For magnetic systems this scale can be the temperature $1 / \beta_{\mathrm{AFM}}\left(k_{B}=\hbar=1\right)$ below which antiferromagnetic (AFM) long-range order manifests itself. In this context, one of the most studied model is perhaps a stacking in three dimensions of chains or square lattices on each of which a nearest-neighbor quantum spin- $S$ Heisenberg model $H_{J}$ with AFM exchange coupling $J>0$ is defined. To model a strong spatial anisotropy, one assumes that there exists a nearest-neighbor AFM exchange coupling $J^{\prime}$ in the directions transverse to the chains or planes that is much weaker than $J, J \gg J^{\prime}>0$. The three-dimensional quantum Hamiltonian is $H_{3 \mathrm{D}}$. Many efforts have been invested for the last 30 years in calculating the $J^{\prime}$ dependence of $1 / \beta_{\mathrm{AFM}}[1-10]$. In this Letter we address the following question: Are there some universal relations that relate $J^{\prime}$ and some observables of $H_{J}$ or $H_{3 \mathrm{D}}$ ?

The motivation for this question comes from the work by Yasuda et al. in Ref. [10] in which the Néel temperature $1 / \beta_{\mathrm{AFM}}$ of $H_{3 \mathrm{D}}$, the $n$-dimensional static staggered susceptibility $\chi_{s}^{(n)}=\chi^{z z}\left(\boldsymbol{Q}, \omega=0 ; \boldsymbol{\beta}_{\mathrm{AFM}}\right)$ of $H_{J}$ where $\boldsymbol{Q}=$ $\pi$ if $n=1$ or $\boldsymbol{Q}=(\pi, \pi)$ if $n=2$, and $J^{\prime} \chi_{s}^{(n)}=1 / \zeta_{n}\left(J^{\prime}\right)$ were computed numerically as a function of $0<J^{\prime} / J \leq 1$. In quasi-two dimensions, it was observed that

$$
J^{\prime} \chi_{s}^{(n=2)}=1 / \zeta_{n=2}
$$

converges to a number as $J^{\prime} \rightarrow 0$ (not $1 / 2$ as predicted by mean-field theory $[1,2])$ that is universal in that it is independent of the magnitude of the spin, including the classi- cal limit $S=\infty$. Although the evidence is less pronounced, the same conclusion was reached in quasi-one dimension.

We want to construct a tractable model that reproduces qualitatively these findings, and we want to understand how these results can be useful to establish experimentally the implied universality. We present a theoretical argument that, as $J^{\prime} / J \downarrow 0$, the function $\zeta_{n=2}$ converges to a constant. Although our scaling argument is too crude to make an estimate for the deviations away from this universal number at finite $J^{\prime} / J$, it suggests that nonuniversal corrections induced by quantum fluctuations are of order $J^{\prime} / J$, whereas those induced by classical fluctuations are of order $1 /\left(J \beta_{\mathrm{AFM}}\right)$. We make the following additional predictions.

First, we consider more general AFM models in the plane, and we consider the case in which, by tuning parameters, it is possible to tune the planar model close to a quantum phase transition, so that the zero-temperature AFM order of the two-dimensional model (without interplane couplings) becomes small. Then, we predict the scaling function

$$
J^{\prime} \chi_{s}^{(2)}=F_{1}\left(c \beta_{\mathrm{AFM}} / \xi^{(2)}\right),
$$

for some scaling function $F_{1}$, in the limit $J^{\prime} / J \downarrow 0$, where $\xi^{(2)}$ is the correlation length of the two-dimensional model at temperature $1 / \beta_{\mathrm{AFM}}$ and $c$ is a spin-wave velocity defined below. Note that in the system considered by Yasuda et al. the planar model is in the renormalized classical regime so that $c \beta_{\mathrm{AFM}} / \xi^{(2)}$ is exponentially small in $c \beta_{\mathrm{AFM}}$ and converges to zero as $J^{\prime} / J \downarrow 0$. Therefore, $F_{1}\left(c \beta_{\mathrm{AFM}} / \xi^{(2)}=0\right)=1 / \zeta_{2}$.

Second, we predict a similar scaling relation that will be valid for quantities which are easier to access experimentally. The susceptibility $\chi_{s}^{(2)}$ defined above is that of the two-dimensional model without the interlayer couplings, and cannot be measured in most real materials. We define $\chi_{\pi, \pi, 0}^{(3)}=\chi^{z z}\left(\pi, \pi, 0, \omega=0 ; \beta_{\mathrm{AFM}}\right)$ to be the static susceptibility in the layered system at wave vector $(\pi, \pi)$ in the plane and wave vector 0 perpendicular to the plane at 
temperature $1 / \beta_{\mathrm{AFM}}$. Then, we predict that

$$
J^{\prime} \chi_{\pi, \pi, 0}^{(3)}=F_{2}\left(c^{(3)} \beta_{\mathrm{AFM}} / \xi_{\pi, \pi, 0}^{(3)}\right),
$$

for some scaling function $F_{2}$, in the limit $J^{\prime} / J \downarrow 0$, where $c^{(3)}$ and $\xi_{\pi, \pi, 0}^{(3)}$ are the in-plane spin-wave velocity and correlation length of $H_{3 \mathrm{D}}$ at temperature $1 / \beta_{\mathrm{AFM}}$ near wave vector $(\pi, \pi, 0)$, respectively.

Third, we predict that similar scaling results hold for other layered models.

In quasi-one dimension [4-9], we expect that similar scaling results will also hold. This does not, however, help us understand the results of Yasuda et al. in quasi-one dimension. The scaling functions $F_{1}, F_{2}$ imply that the classical and quantum models will show the same $\zeta^{(1)}$ only if $c \beta_{\mathrm{AFM}} \ll \xi^{(1)}$. However, as the one-dimensional Heisenberg model is instead quantum critical, there is a universal $\zeta^{(1)}$ for the half-integer spin chain, which need not be the same as that for the integer or classical spin chains. Thus, the one-dimensional results remain a puzzle; however, additional simulations on the $S=1$ chain do show deviations in $\zeta^{(1)}$ for sufficiently small $J^{\prime} / J$ [11], and further simulations may show a difference between the half-integer and classical systems.

Physical motivation. - Here, we present a physical motivation for the results above and a brief microscopic derivation of the relevant nonlinear sigma model. In the next section, we show these scaling results using a renormalization group (RG) for this nonlinear sigma model. The reason we use this model is that we want to illustrate the effects of field renormalization, and the nonlinear sigma model RG already has a nontrivial field renormalization at leading order in the coupling constant, while such a renormalization is not seen until order $\epsilon^{2}(1 / N)$ in a $4-\epsilon$ (large $N$ ) expansion.

Since the interplane interaction is weak, we can treat it perturbatively at the microscopic level. Following standard steps, in the absence of the interplane interaction, we can first derive the partition function for the two-dimensional $O(N)$ quantum nonlinear sigma model (2D QNLSM) with field $\boldsymbol{n}_{k}(\boldsymbol{r}, \boldsymbol{\tau})$, where $k$ is a discrete index labeling individual planes, $\boldsymbol{r}$ is a two-dimensional vector describing coordinates in the plane, and $\tau$ is imaginary time. The relevant action for plane $k$ is $S_{k}=S_{k}^{(1)}+S_{k}^{(2)}$ where

$$
\begin{aligned}
S_{k}^{(1)} & :=\int \mathcal{L}_{k}^{(1)} \equiv \int_{0}^{\beta} d \tau \int_{a}^{L} d^{2} \boldsymbol{r} \frac{c}{2 a g}\left(\partial_{\mu} \boldsymbol{n}_{k}\right)^{2}, \\
S_{k}^{(2)} & :=\int \mathcal{L}_{k}^{(2)} \equiv-\int_{0}^{\beta} d \tau \int_{a}^{L} d^{2} \boldsymbol{r} \frac{c}{a^{3}} Z_{h} \boldsymbol{h} \cdot \boldsymbol{n}_{k} .
\end{aligned}
$$

Here, the lattice spacing $a$ plays the role of the microscopic ultraviolet cutoff, i.e., $\Lambda \sim 1 / a$ that of an upper cutoff on momenta. The linear size $L$ of the plane is the largest length scale of the problem. The derivative $\partial_{\mu}=\left(\partial_{c \tau}, \nabla\right)$ depends on the spin-wave velocity $c$ in the plane which is of order $J a$. The dimensionless coupling constant $g$ de- pends on the microscopic details of the intraplane interactions. The dimensionless background field $\boldsymbol{h}$, where $h=|\boldsymbol{h}|$, is the external source for a static staggered magnetic field conjugate to the planar AFM order parameter of the underlying lattice model. It breaks the $O(N)$ symmetry of Lagrangian (4a) down to $O(N-1)$ and as such acts as an infrared regulator. The dimensionless coupling $Z_{h}$ is the field renormalization constant associated with $\boldsymbol{n}_{k}$. The use of the continuum limit within each of the planes labeled by $k$ is justified if we are after the physics on length scales much longer than $a$.

The interplane nearest-neighbor AFM coupling $J^{\prime}$ gives the characteristic interplane spin-wave velocity $c^{\prime} \sim J^{\prime} a$ and length scale $a^{\prime} \equiv\left(J / J^{\prime}\right)^{1 / 2} a$. The couplings $J^{\prime}$, $g$ get renormalized as discussed below, so the velocity $c^{\prime}$ changes at longer length scales. For a very weak nearest-neighbor interplain AFM coupling, $J^{\prime} \ll J$, the physics on length scales much larger than $a$ but yet not much larger than $a^{\prime}$ is captured by the partition function

$$
\begin{aligned}
Z & =\int_{\mathbb{R}^{N}}\left[\prod_{k} \mathcal{D}\left[\boldsymbol{n}_{k}\right] \delta\left(\boldsymbol{n}_{k}^{2}-1\right)\right] \exp \left(-\sum_{k} \int \mathcal{L}_{k}\right), \\
\mathcal{L}_{k} & =\mathcal{L}_{k}^{(1)}+\mathcal{L}_{k}^{(2)}+\mathcal{L}_{k}^{(3)} .
\end{aligned}
$$

The Lagrangian $\mathcal{L}_{k}^{(3)}$ that encodes the effect of the microscopic nearest-neighbor interplane AFM interaction $J^{\prime}$ is approximated by

$$
\mathcal{L}_{k}^{(3)}=\frac{J^{\prime} Z^{\prime}}{2 a^{2}}\left(\boldsymbol{n}_{k}-\boldsymbol{n}_{n+1}\right)^{2},
$$

where $Z^{\prime}$ renormalizes as $Z_{h}^{2}$ to lowest order in $J^{\prime} / J$,

$$
Z^{\prime}=Z_{h}^{2}\left[1+\mathcal{O}\left(J^{\prime} / J\right)\right] .
$$

This defines the so-called three-dimensional strongly anisotropic $O(N)$ QNLSM.

Renormalization group.-Here, we present an RG analysis of the nonlinear sigma model (5). The most important result in this section is that the identity (6) is preserved under the RG flow up to the length scale at which the scale dependent effective anisotropy (13) is of order 1.

We perform a RG analysis following Polyakov for convenience [12]. In each plane labeled by $k$, we write

$$
\boldsymbol{n}_{k}=\boldsymbol{m}_{k}\left(1-\phi_{k}^{2}\right)^{1 / 2}+\sum_{a=1}^{N-1} \boldsymbol{e}_{k}^{a} \boldsymbol{\phi}_{k}^{a}
$$

The field of unit length $\boldsymbol{m}_{k}$ encodes the planar AFM order expected in the limit $g / c \downarrow 0$, while the $N-1$ fields $\boldsymbol{e}_{k}^{a}$ capture the deviations away from the direction $\boldsymbol{m}_{k}$ of symmetry breaking, i.e., the $N-1 \boldsymbol{e}_{k}^{a}$ form an orthonormal basis of vectors orthogonal to $\boldsymbol{m}_{k}$. The $N-1$ coefficients $\phi_{k}^{a}$ make up the vector $\phi_{k}$. To leading order in an expansion in powers of $g / c$ of the parametrization (7), the field $\boldsymbol{m}_{k}$ is the slow mode while the $N-1$ fields $\phi_{k}^{a}$ represent 
fast modes with characteristic 2-momenta $\tilde{\Lambda}<|p| \leq \Lambda$. Substituting Eq. (7) into Eq. (5) gives the Lagrangian

$$
\begin{aligned}
\mathcal{L}_{k}^{(1)}+\mathcal{L}_{k}^{(2)}= & \frac{c}{2 a g}\left[\left(\partial_{\mu} \phi_{k}^{a}-A_{k \mu}^{a b} \phi_{k}^{b}\right)^{2}+\left(B_{k \mu}^{a}\right)^{2}\right. \\
& \left.+B_{k \mu}^{a} B_{k \mu}^{b}\left(\phi_{k}^{a} \phi_{k}^{b}-\phi_{k}^{2} \delta^{a b}\right)\right] \\
& -\frac{c}{a^{3}} Z_{h} \boldsymbol{h} \cdot \boldsymbol{m}_{k}\left(1-\phi_{k}^{2}\right)^{1 / 2}
\end{aligned}
$$

to leading order in an expansion in powers of $g / c$. The $N-$ 1 coefficients $B_{k \mu}^{a}$ are defined by $\partial_{\mu} \boldsymbol{m}_{k}=\sum_{a=1}^{N-1} B_{\mu}^{a} \boldsymbol{e}_{k}^{a}$. The $(N-1)(N-2) / 2$ independent coefficients $A_{k \mu}^{a b}=$ $-\left(\partial_{\mu} \boldsymbol{e}_{k}^{b}\right) \cdot \boldsymbol{e}_{k}^{a}$. The RG flows of the dimensionless couplings $g, Z_{h} h$, and $t \equiv 1 /(J \beta)$ that follow after integration over the fast modes $\phi_{k}$ in the limit of no interplane interactions were computed by Chakravarty, Halperin, and Nelson to leading order in $g / c$ (see Fig. 1) [13]. To this order, $c$ is unchanged.

To quantify the very weak microscopic interplanar coupling, we define the anisotropy $\tilde{\alpha}$ as the ratio of the importance of $\mathcal{L}_{k}^{(1)}$ to $\mathcal{L}_{k}^{(3)}$ when the upper cutoff on the momenta is $\tilde{\Lambda}$. By assumption, this anisotropy is strong at the microscopic level (upper cutoff $\Lambda$ ),

$$
\alpha=g J^{\prime} Z^{\prime} / J \ll 1 .
$$

Next, we consider the renormalization of $\mathcal{L}_{k}^{(1)}$ in Eq. (8) when $\boldsymbol{h}=\mathbf{0}$ and of the interplane interaction

$$
\begin{aligned}
\mathcal{L}_{k}^{(3)}= & \frac{J^{\prime} Z^{\prime}}{2 a^{2}}\left[\left(1-\phi_{k}^{2}\right)^{1 / 2} \boldsymbol{m}_{k}-\left(1-\phi_{k+1}^{2}\right)^{1 / 2} \boldsymbol{m}_{k+1}\right. \\
& \left.+\sum_{a=1}^{N-1}\left(\phi_{k}^{a} \boldsymbol{e}_{k}^{a}-\phi_{k+1}^{a} \boldsymbol{e}_{k+1}^{a}\right)\right]^{2}
\end{aligned}
$$

after averaging over the fast modes $\phi_{k}$. To this end, we introduce the renormalized values
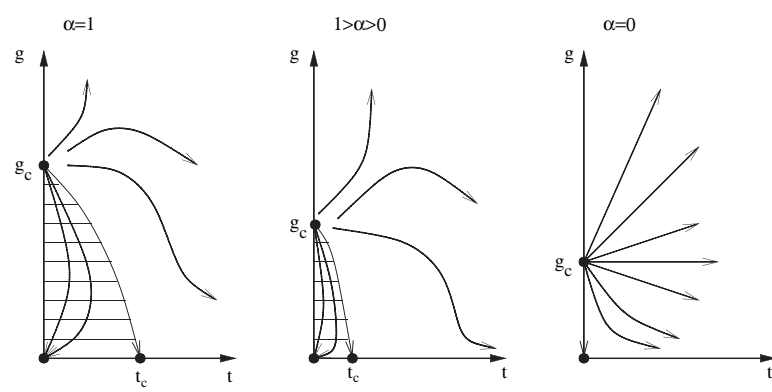

FIG. 1. Phase diagram and RG flows for the 3D QNLSM ( $\alpha=$ $1)$ and 2D QNLSM $(\alpha=0)$ after Ref. [13]. Conjectured phase diagram and RG flow for the anisotropic 3D QNLSM $(1>\alpha>$ $0)$. The shaded regions have long-range Néel order. The figure for $1>\alpha>0$ shows a two-dimensional slice of the threedimensional RG flow, as $\tilde{\alpha}$ is changing under this flow.

$$
\begin{aligned}
\frac{1}{\tilde{g}} & =\frac{1}{g}\left(\frac{\Lambda}{\tilde{\Lambda}}\right)\left(1+\left\langle\phi_{k}^{a} \phi_{k}^{b}-\phi_{k}^{2} \delta^{a b}\right\rangle\right), \\
\frac{1}{\tilde{t}} & =\frac{1}{t}\left(1+\left\langle\phi_{k}^{a} \phi_{k}^{b}-\phi_{k}^{2} \delta^{a b}\right\rangle\right), \\
\tilde{Z}_{h} & =Z_{h}\left(1-\frac{1}{2}\left\langle\phi_{k}^{2}\right\rangle+\cdots\right),
\end{aligned}
$$

at the scale $\tilde{\Lambda}$ as a result of averaging over the fast modes $\phi_{k}$. For $\alpha \ll 1$, this average over fast modes is $\left\langle\phi_{k}^{a} \phi_{l}^{b}\right\rangle=$ $\delta_{k l} \delta^{a b} \ln (\Lambda / \tilde{\Lambda}) \frac{g}{4 \pi} \operatorname{coth}(g / 2 t)$. Furthermore,

$$
\mathcal{L}_{k}^{(3)} \approx \frac{J^{\prime} Z^{\prime}}{2 a^{2}} \frac{\tilde{Z}_{h}^{2}}{Z_{h}^{2}}\left(\boldsymbol{m}_{k}-\boldsymbol{m}_{k+1}\right)^{2}
$$

from which follows the renormalizations

$$
\tilde{\alpha}=\left(\frac{\Lambda}{\tilde{\Lambda}}\right)^{3} \frac{\tilde{g} \tilde{Z}^{\prime}}{g Z^{\prime}} \alpha, \quad \frac{\tilde{Z}^{\prime}}{Z^{\prime}}=\frac{\tilde{Z}_{h}^{2}}{Z_{h}^{2}},
$$

so long as $\tilde{\alpha} \ll 1$.

Let us follow the RG flows encoded by Eqs. (11) and (13) starting from the initial values $g>0, t \equiv$ $1 /\left(J \beta_{\mathrm{AFM}}\right)<\infty$, and $1 \gg \alpha>0$ [see Eq. (9)] corresponding to a point on the phase boundary between the Néel and paramagnetic phase (see Fig. 1). Aside from the thermal de Broglie wavelength of the spin waves $c \beta_{\mathrm{AFM}}$, the initial values $g$ and $t$ define a second characteristic length scale, the correlation length $\xi^{(2)}$ in the 2D QNLSM, in view of $1 \gg \alpha>0$. We distinguish two cases. In the renormalized classical regime $c \beta_{\mathrm{AFM}} / \xi^{(2)} \ll 1$. In the quantum critical regime $c \beta_{\mathrm{AFM}} / \xi^{(2)} \sim 1$. Finally, we denote by $\xi_{\text {cross }}$ the RG length scale at which $\tilde{\alpha} \sim 1$ and beyond which the RG flows Eqs. (11) and (13) should be replaced by the flows of the isotropic 3D QNLSM; naive scaling gives $\xi_{\text {cross }} \sim a^{\prime}$, but the RG flows above will change this scaling. Any two of these characteristic length scales, $c \beta_{\mathrm{AFM}}, \xi^{(2)}$, and $\xi_{\text {cross }}$, fix the third one since the RG flows are constrained to the boundary between the Néel and paramagnetic phases by assumption. Without loss of generality, we consider the case $\Lambda>1 /\left(c \beta_{\mathrm{AFM}}\right)$. As we lower the upper momentum cutoff, the RG scale $\tilde{\Lambda}^{-1}$ will eventually become larger than $c \beta_{\mathrm{AFM}}$. We consider RG scales $\tilde{\Lambda}^{-1} \gg c \beta_{\mathrm{AFM}}$, for which the quantum fluctuations are important.

We are after the quantity $J^{\prime} \chi_{s}^{(2)}$ when $J^{\prime} \ll J$. By dimensional analysis and for RG length scale $\tilde{\Lambda}^{-1} \geq \xi^{(2)}$ $\chi_{s}^{(2)} \sim\left(\Lambda \xi^{(2)}\right)^{2} \tilde{Z}_{h}^{2} \beta_{\mathrm{AFM}}$. We claim that $J^{\prime} \chi_{s}^{(2)} \sim 1$ up to nonuniversal corrections of order $J^{\prime} / J$ and $\left(J \beta_{\mathrm{AFM}}\right)^{-1}$ as a consequence of the facts that $\tilde{Z}_{h}^{2}$ can be estimated from the approximate "Ward identity" $\tilde{Z}_{h}^{2} \sim \tilde{Z}^{\prime}$ and $J^{\prime}$ can be estimated from the condition $\tilde{\alpha} \sim 1$. The proof begins with the flow $\tilde{\alpha} \sim(\Lambda / \tilde{\Lambda})^{2} \alpha$ as long as $\tilde{\Lambda}^{-1} \lesssim c \beta_{\mathrm{AFM}}$. Beyond the RG length scale $\tilde{\Lambda}^{-1} \sim c \beta_{\mathrm{AFM}}$, we can replace the $2 \mathrm{D}$ QNLSM in each plane by a classical 2D NLSM with the effective coupling $\tilde{g}_{\mathrm{cl}}$, where $\tilde{g}_{\mathrm{cl}}=\tilde{g}$ at the upper momentum cutoff $1 /\left(c \beta_{\mathrm{AFM}}\right)$. If so, the effective anisotropy con- 
tinues to decrease as $\tilde{\alpha}=(\Lambda / \tilde{\Lambda})^{2} \tilde{g}_{\mathrm{cl}} \tilde{Z}^{\prime} J^{\prime} \beta_{\mathrm{AFM}}$ continues to grow until it reaches the isotropic RG scale $\tilde{\alpha} \sim 1$ at which $\tilde{g}_{\mathrm{cl}} \sim 1$. Solving for $J^{\prime}$ in terms of the renormalized parameters when $\tilde{\alpha} \sim 1$, we arrive at Eq. (1) in the renormalized classical regime.

Note that each of these relations, such as $\tilde{\alpha} \sim 1$ and $\tilde{g}_{\mathrm{cl}} \sim 1$, is defined up to a multiplicative constant that depends on the details of how we define the RG. However, the dimensionless combination in Eq. (1) is universal. The reason for the universality is that all the microscopic details of the Heisenberg model are encoded into the three independent quantities $\tilde{g}, \tilde{Z}^{\prime}$, and $\tilde{Z}_{h}$ on any length scale much larger than $a$. Let us perform the RG flow to some scale such that $\tilde{\alpha}$ is much less than unity. Then, the identity (6) relates $\tilde{Z}^{\prime}$ to $\tilde{Z}_{h}$, leaving only two quantities independent in the classical regime, say $\tilde{g}_{\mathrm{cl}}$ and $\tilde{\alpha}$. The requirement of criticality relates $\tilde{g}_{\mathrm{cl}}$ to $\tilde{\alpha}$, leaving only one independent quantity, say $\tilde{\alpha}$. Choosing the renormalization scale to be some given fraction of the correlation length in the two-dimensional model fixes the last quantity, and thus there are no independent parameters left.

Near a quantum critical point and as is the case for the renormalized classical regime, the length scale at which $\tilde{\alpha} \sim 1$ is of the order $\xi^{(2)}$. Now, however, there is no significant separation of scales between $c \beta_{\mathrm{AFM}}$ and $\xi^{(2)}$ anymore, i.e., $\tilde{\alpha} \sim 1$ already at $c \beta_{\mathrm{AFM}}$. Correspondingly, there will be universal corrections to (6) in the form $\tilde{Z}^{\prime} / Z^{\prime}=\kappa(1, g(1)) \tilde{Z}_{h}^{2} / Z_{h}^{2}$ where the function $\kappa$ of $\alpha$ and $g$ is universal with $\kappa(0, g)=1$. The deviations in the quantum critical regime from the limiting value of $J^{\prime} \chi_{s}^{(2)}$ in the classical renormalized regime define the universal scaling function $F_{1}$ of $c \beta_{\mathrm{AFM}} / \xi^{(2)}$.

Similarly, the correlation at the $(\pi, \pi, 0)$ point is of order $\tilde{\Lambda}^{-1}$ in the plane while it is of order a single interlayer spacing between the planes. Thus, $\chi_{\pi, \pi, 0}^{(3)} \sim \chi_{s}^{(2)}$ and $\xi_{\pi, \pi, 0}^{(3)} \sim \xi^{(2)}$, and so Eq. (3) follows.

We close by noting that all arguments presented here for a nonlinear sigma model with $O(N)$ symmetry extend to nonlinear sigma models defined on Riemannian manifolds with a positive curvature tensor. For example, we expect similar universal scaling relations for a stacking of AFM Heisenberg models on a triangular lattice.

Discussion. - There is a large amount of published data on quasi-two-dimensional antiferromagnets such as the spin-1/2 compounds $\mathrm{La}_{2} \mathrm{CuO}_{4}$ [14,15], $\mathrm{Sr}_{2} \mathrm{CuO}_{2} \mathrm{Cl}_{2}$ [16], and copper formate tetradeuterate (CFTD) [17], as well as other higher-spin compounds. In this body of work, the dependence on temperature of a number $S_{0 \beta}^{(\text {a.u.) }}$ measured in arbitrary units is believed to give a good approximation to the instantaneous structure factor $S(\pi, \pi, 0, t=0 ; \beta)$ [18] in a regime of temperatures above $1 / \beta_{\mathrm{AFM}}$ for which spin fluctuations are predominantly isotropic in spin space. In this context, we note that the product $J^{\prime} \beta_{\mathrm{AFM}} S(\pi, \pi, 0, t=$ $\left.0 ; \beta_{\mathrm{AFM}}\right)$ should also obey a scaling law of the form (3) for some scaling function $F_{3}$ that depends on the universality class of the transition at the ordering wave vector; we note that in all cases, the universality class at $\beta_{\mathrm{AFM}}$ is Ising or $X Y$ rather than Heisenberg. For all these compounds, $J^{\prime} / J$ is very small, less than $10^{-4}$. Above the critical temperature, this number $J^{\prime} \beta S_{0 \beta}$ decreases. After converting the arbitrary units of the measured $S_{0 \beta}^{(\text {a.u.) }}$ by multiplication of the number $S_{0 \beta^{*}}^{\text {(m.c.) }} / S_{0 \beta^{*}}^{\text {(a.u. }}$, where $1 / \beta^{*}$ is the measured temperature at which the correlation length in units of the lattice spacing is 3 and $S_{0 \beta^{*}}^{\text {(m.c.) }}$ is borrowed from the Monte Carlo simulation by Kim and Troyer in Ref. [19], we find that $J^{\prime} \beta S_{0 \beta}$ takes the values 1.17, 0.001, and 0.077, at the temperatures $T=337,278$, and $18 \mathrm{~K}$ that are 12 , 21.5 , and $1.5 \mathrm{~K}$ above the corresponding ordering temperatures of $\mathrm{La}_{2} \mathrm{CuO}_{4}, \mathrm{Sr}_{2} \mathrm{CuO}_{2} \mathrm{Cl}_{2}$, and CFTD, respectively. These are the lowest temperatures for which we have data within the Heisenberg universality class. This result is largely in accord with our predictions: in $\mathrm{La}_{2} \mathrm{CuO}_{4}$, the extrapolated data show only a small increase in $S_{0 \beta}$ from $T=337 \mathrm{~K}$ to $T=325 \mathrm{~K}$, and hence $J^{\prime} \beta_{\mathrm{AFM}} S\left(\pi, \pi, 0, t=0 ; \beta_{\mathrm{AFM}}\right)$ will indeed be of order unity, while in the other compounds $S_{0 \beta}$ shows a very rapid increase approaching the ordering temperature. Thus, a measurement of $S\left(\pi, \pi, 0, t=0 ; \beta_{\mathrm{AFM}}\right)$ at the ordering temperature in absolute units would be very desirable.

We thank M. Troyer for explaining [10] and H.M. Ronnow for useful discussions. This work was supported by DOE Grant No. W-7405-ENG-36.

*Electronic address: hastings@cnls.lanl.gov

[1] D. J. Scalapino et al., Phys. Rev. B 11, 2042 (1975).

[2] H. J. Schulz, Phys. Rev. Lett. 77, 2790 (1996).

[3] V. Yu. Irkhin and A. A. Katanin, Phys. Rev. B 55, 12318 (1997); 57, 379 (1998); V. Yu. Irkhin, A. A. Katanin, and M. I. Katsnelson, ibid. 60, 1082 (1999).

[4] I. Affleck et al., J. Phys. A 27, 7313 (1994).

[5] I. Affleck and B. I. Halperin, J. Phys. A 29, 2627 (1996).

[6] F. H. L. Essler et al., Phys. Rev. B 56, 11001 (1997).

[7] Z. Wang, Phys. Rev. Lett. 78, 126 (1997).

[8] V. Yu. Irkhin and A. A. Katanin, Phys. Rev. B 61, 6757 (2000).

[9] M. Bocquet, Phys. Rev. B 65, 184415 (2002).

[10] C. Yasuda et al., Phys. Rev. Lett. 94, 217201 (2005).

[11] M. Troyer (private communication).

[12] A. M. Polyakov, Phys. Lett. 59B, 79 (1975).

[13] S. Chakravarty et al., Phys. Rev. B 39, 2344 (1989).

[14] R. J. Birgeneau et al., Phys. Rev. B 59, 13788 (1999).

[15] A. M. Toader et al., Phys. Rev. Lett. 94, 197202 (2005).

[16] M. Greven et al., Z. Phys. B 96, 465 (1995).

[17] H. M. Ronnow et al., Phys. Rev. Lett. 82, 3152 (1999).

[18] R. J. Birgeneau, J. Skalyo, and G. Shirane, Phys. Rev. B 3, 1736 (1971).

[19] J.-K. Kim and M. Troyer, Phys. Rev. Lett. 80, 2705 (1998). 